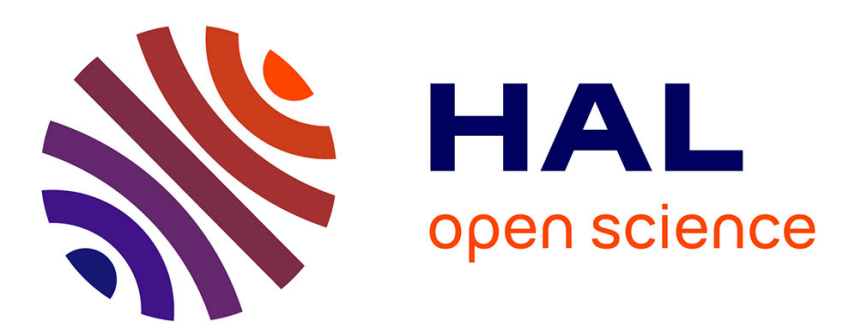

\title{
Biomolecular hydration dynamics: a jump model perspective
}

Aoife C Fogarty, Elise Duboué-Dijon, Fabio Sterpone, James T. Hynes, Damien Laage

\section{- To cite this version:}

Aoife C Fogarty, Elise Duboué-Dijon, Fabio Sterpone, James T. Hynes, Damien Laage. Biomolecular hydration dynamics: a jump model perspective. Chemical Society Reviews, 2013, 42 (13), 10.1039/C3CS60091B . hal-01774658

\section{HAL Id: hal-01774658 \\ https://hal.sorbonne-universite.fr/hal-01774658}

Submitted on 23 Apr 2018

HAL is a multi-disciplinary open access archive for the deposit and dissemination of scientific research documents, whether they are published or not. The documents may come from teaching and research institutions in France or abroad, or from public or private research centers.
L'archive ouverte pluridisciplinaire HAL, est destinée au dépôt et à la diffusion de documents scientifiques de niveau recherche, publiés ou non, émanant des établissements d'enseignement et de recherche français ou étrangers, des laboratoires publics ou privés. 


\title{
Biomolecular hydration dynamics:
}

\section{a jump model perspective}

\author{
Aoife C. Fogarty* Elise Duboué-Dijon ${ }^{\dagger} \quad$ Fabio Sterpone ${ }^{\ddagger}$ \\ James T. Hynes ${ }^{\S}$ Damien Laage
}

7 July 2013

\begin{abstract}
The dynamics of water molecules within the hydration shell surrounding a biomolecule can have a crucial influence on its biochemical function. Characterizing their properties and the extent to which they differ from those of bulk water have thus been long-standing questions. Following a tutorial approach, we review the recent advances in this field and the different approaches which have probed the dynamical perturbation experienced by water in the vicinity of proteins or DNA. We discuss the molecular factors causing this perturbation, and describe how they change with temperature. We finally present more biologically relevant cases beyond the dilute aqueous situation. A special focus is on the jump model for water reorientation and hydrogen bond rearrangement.
\end{abstract}

${ }^{*}$ Department of Chemistry, UMR ENS-CNRS-UPMC-8640, Ecole Normale Supérieure, rue Lhomond, Paris, France

${ }^{\dagger}$ Department of Chemistry, UMR ENS-CNRS-UPMC-8640, Ecole Normale Supérieure, rue Lhomond, Paris, France

${ }^{\ddagger}$ Laboratoire de Biochimie Théorique, CNRS, UPR9080, Univ. Paris Diderot, Sorbonne Paris Cité, 13 rue Pierre et Marie Curie, 75005, Paris, France

${ }^{\S}$ Department of Chemistry, Ecole Normale Supérieure, Paris, France and University of Colorado, Departement of Chemistry and Biochemistry, Boulder, Colorado, USA

I Department of Chemistry, UMR ENS-CNRS-UPMC-8640, Ecole Normale Supérieure, rue Lhomond, Paris, France, E-mail: damien.laage@ens.fr 


\section{Key Learning Points}

- Why is water dynamics next to a biomolecule important for its biochemical function?

- How can the hydration shell be defined? How thick is it?

- Is the biomolecular hydration layer viscous or labile?

- How heterogeneous is water dynamics within the shell?

- What is the molecular origin of the dynamical perturbation induced by the biomolecule on water? chemistry vs. topology

- How does the dynamical perturbation change with temperature?

- Can one go beyond the dilute globular protein case?

\section{Introduction}

Classic biochemistry textbooks usually represent biomolecules as static and isolated structures. It is now being increasingly realized that this picture is oversimplified and ignores some essential aspects. In biologically relevant environments, a biomolecule is immersed in an aqueous solvent which affects its properties, including its flexibility to undergo transitions between different conformations which are crucial for its biochemical functioning. A three-dimensional network of water molecules connected together via hydrogen $(\mathrm{H})$-bonds surrounds the biomolecule. Due to the great disparity between the masses of a water molecule and of a biological macromolecule like a protein or a DNA oligomer, water dynamics in this network is usually much faster than that of the biomolecule. Key motions of these water molecules include exchanges of H-bond partners which modify the connectivity of the H-bond network and reorient the water permanent dipole, thus changing e.g. the electrostatic interaction with the biomolecule. The typical timescale of these motions lies in the picosecond $\left(10^{-12} \mathrm{~s}\right)$ range; in strong 
contrast, typical tumbling motions and large-amplitude conformational motions of proteins are in the nanosecond $\left(10^{-9} \mathrm{~s}\right)$ range and beyond, i.e. more than 1,000 times slower.

Due to their important biochemical implications, the properties of biomolecular hydration shells and their impact on biochemical function have been much studied both experimentally and theoretically through a wide range of techniques. Many excellent reviews on different aspects of biomolecular hydration shells already exist, ${ }^{1-7}$ some of them by groups which have pioneered the field. Here we focus on the dynamics of water next to a biomolecule and more specifically on how and how much the dynamics is perturbed by the biomolecule's presence. Many other important aspects of biomolecular hydration shells will be mentioned only very briefly, including e.g. the reciprocal action of the hydration layer on the biomolecule's function, the thermodynamic properties of water molecules next to a protein or DNA, and the details of the many techniques which have been applied to this problem. Our present goal is the limited one of providing a short tutorial review to this very active field. We immediately stress that it is necessarily partial and subjective, and the limited number of references did not allow us to do justice to all groups working in the field. Nonetheless, while we focus on questions where our group has made some contributions, we also attempt to provide the reader with a broader perspective. The rapid progress in this field has greatly benefited from the fruitful confrontation of ideas, techniques and models, and we try here to identify what is now generally agreed upon and what is still debated. A larger fraction of our discussion will be focused on proteins because DNA hydration dynamics has been so far less studied, but the same general principles apply to both types of systems.

This review is structured around a series of simple questions of increasing complexity. We first illustrate the biochemical importance of hydration dynamics through a few examples. We then successively examine the hydration layer's definition and whether it is labile or viscous. We subsequently describe the dynamical heterogeneity within the shell and discuss the origin of the perturbation of the shell together with its temperature dependence. We finally consider how the concepts developed for biomolecules 
in dilute solution can be extended to more complex but also more biologically relevant situations and finally we offer some concluding remarks.

\section{Why is water dynamics next to a biomolecule impor- tant for its biochemical function?}

Hydration shell dynamics has been suggested to play a prominent role in a wide range of biochemical processes. ${ }^{7}$ This view highlights for us the necessity to acquire a deeper understanding of the influences of biomolecules and their aqueous environment on each other. In fact, this notion of water's importance in a biochemical context is of fairly recent vintage. In the not-so-distant past, water was frequently rather neglected in studies of biomolecular systems, the idea being that it served as merely the 'spectator' solvent for biomolecules whose intrinsic and interesting properties were independent of hydration-shell structure or dynamics. However, it has now become quite clear that water is not only the natural environment for biochemical processes, it is also often an active and even key participant.

Accordingly, it is now widely accepted that the inclusion of the biomolecular hydration shell in the study of proteins and nucleic acids is crucial for a complete understanding of their function. The structural and thermodynamic properties of the hydration shell are key factors e.g. to ensure the biomolecule's structural stability and in processes like molecular recognition and ligand binding. ${ }^{8,9}$ Here, our focus is rather on dynamical aspects, which also play a prominent role in essential aspects of biomolecular function. We now briefly review some important examples of this.

Enzyme catalysis. The catalytic activity of enzymes drops dramatically when their hydration level is decreased. ${ }^{10,11}$ This has been measured e.g. in mixtures of water with an increasing fraction of aprotic organic cosolvent. ${ }^{10}$ The traditional explanation assigns the plunge in enzymatic activity to an enhanced protein rigidity. ${ }^{10}$ When water is gradually removed, the protein replaces by intramolecular bonds the H-bonds formerly existing with water. This decreases the protein's flexibility and hinders the conformational transitions occurring during the catalytic transformation, leading to a 
decreased activity. The situation is quite different when water is present; the great lability of the hydration layer, i.e. the highly dynamic nature of the water H-bond network surrounding the protein, is suggested to act as a lubricant of these conformational transitions. Support for this view is provided by recent spectroscopy experiments which have found a correlation between water dynamics and the kinetics of local active-site rearrangements during catalysis. ${ }^{12}$ However, while picturing water as a lubricant of enzyme catalysis has a seductive appeal, a rigorous molecular definition of this effect is still missing, and enzyme-specific effects probably also contribute. In addition, whether the origin is dynamical or thermodynamical for these and other phenomena is not clearly established. For example, is the catalytic influence due to time scaledependent features of the environment such as dielectric relaxation times or viscosity or is it solely one of alteration of the activation free energy ? This aspect is for example related to the current debate on the importance of dynamical effects in enzyme catalysis. ${ }^{13}$

Protein/Drug Binding to DNA. Water has been implicated in several aspects of binding to DNA. For example, the release of water in DNA's minor groove has been suggested to provide an entropic driving force for the binding of proteins, ${ }^{6}$ while for assorted drugs, ligand-water-DNA bridges have been argued to enable overcoming of 'fit' problems in the DNA grooves. ${ }^{14}$ Finally, in a more clearly dynamical vein, water hydration shell rearrangement has been implicated as a key ingredient for the intercalation (insertion) of anti-cancer drugs into the DNA minor groove. ${ }^{15}$

Heat protection. Most biomolecules are only active in one specific structure, e.g. the native fold for proteins. But these structures are extremely fragile and can be easily denatured, since their delicate scaffolding relies on H-bonds whose dissociation free energies are only a few $\mathrm{kcal} / \mathrm{mol}$. As is well known, the double strand helix of DNA is held together by H-bonds between pairs of nucleic acids. When a DNA molecule is electronically excited by a UV photon, the electronic excitation is quickly transformed into a vibrational excitation; however this excess energy is still potentially lethal for the DNA structure and must be quickly dissipated to avoid disaster. This is where the hydration layer dynamics can play an important protective role. The numerous 
vibrational modes available in the solvent can accept this excess energy and preserve the biomolecule from structural degradation. ${ }^{16}$

Protein folding and aggregation. Protein folding in water is thermodynamically driven by hydrophobic interactions which favor the folded conformation where, phrased very schematically, the hydrophobic protein core is protected from water. It has been suggested that the dynamic rearrangement of the water solvent, which has to be expelled from the protein core, makes an important contribution to the free energy barrier and thus the protein folding kinetics. ${ }^{6}$ Anomalously fast water dynamics next to key residues is proposed to facilitate the formation of aggregates called amyloids which are involved in neurodegenerative diseases such as Alzheimer's and Parkinson's. ${ }^{17}$

Redox centers and proton pumps. A last example can be found in electron and proton transfer reactions in proteins, where water dynamics is an important part of the mechanism. In electron transfers between protein redox centers, e.g. in the respiratory chain and in photosynthesis, the transfer rate constant was suggested to be governed by large fluctuations in the hydration shell which lead to dielectric relaxation. ${ }^{18}$ In proton pumps such as bacteriorhodopsin, long-distance proton transport within the protein is mediated by a chain of water molecules whose dynamics is critical for a fast and controlled transfer of the proton. ${ }^{19}$

\section{How can the hydration shell be defined? How thick is it?}

The idea of a hydration layer surrounding a biomolecule is an intuitive concept, and one pictures a sheath made of water molecules and covering the biomolecule. However, when a rigorous and specific definition is needed, ambiguities arise. How thick is this layer? Is the thickness the same everywhere?

We first stress that we are focusing our attention on the labile hydration shell, and not on the few long-lived internal water molecules, which are deeply buried within cavities and whose escape dynamics usually occur on a timescale of nanoseconds or more. 
In molecular simulations like molecular dynamics and Monte Carlo, a convenient and widely employed definition relies on geometric criteria. For example, one can select all those water molecules whose oxygen lies within a given, maximum distance from the closest biomolecule atom. This maximum distance is usually taken to include the first layer of water molecules (typically $3.5 \AA$ ), which is close to the intuitive picture. It can however also be varied with the nature of the closest biomolecule site. The idea here is to account for the different interaction strengths of water with different chemical groups: hydrophobic groups tend to repel water molecules while polar groups tend to attract them. Other, more sophisticated, definitions exist. One such definition is based on a Voronoi tesselation of space, which assigns to each (heavy) atom a polyhedron which includes all points in space closer to it than to any other site. All the water polyhedra in contact with a biomolecule polyhedron are then considered to be in the first shell (see e.g. ${ }^{20,21}$ ).

Unfortunately, no such geometric selection is possible experimentally, since the signal is collected from the entire sample. In dilute solutions, the signal is thus usually dominated by bulk water, making it very difficult to isolate the part coming from the small fraction of water molecules located next to the biomolecule. The properties of these interfacial water molecules differ from those in the bulk, and the biomolecule's influence can be treated as a perturbation. The shell is then usually defined in a perturbative way: it includes all water molecules whose dynamics is affected by the biomolecule's presence. We now briefly review the key techniques employed to measure the perturbation's intensity and discuss for each of them which water molecules are probed.

A first group of techniques, including e.g. NMR, THz and neutron spectroscopies, directly probe the dynamics of water molecules. The collected signal is averaged over all water molecules within the sample; as a result, a single measurement cannot discriminate between a situation with a small hydration shell with strongly retarded dynamics and another with a moderate slowdown affecting many water molecules. Several measurements at different solute concentrations are therefore necessary and the results are interpreted within a two-state model. In this model, the average dynamics 
within the sample is a population-weighted average over the hydration shell and bulklike states, and the water dynamics within each state is assumed to be concentrationindependent. Among these techniques, NMR probes the reorientation dynamics of individual water molecules and employs dilute aqueous solutions where the two-state picture is reasonable. The only additional information required is the number of water molecules affected by a biomolecule, which is estimated separately, for example through molecular dynamics or solvent-accessible surface area calculations. For the typical motions probed by NMR, e.g. the reorientation of a water molecular vector (or tensor for the isotopic variant ${ }^{17} \mathrm{OH}_{2}$ ), the perturbation induced by the solute is short-ranged and usually considered to affect only the first layer of water molecules next to the biomolecular interface (i.e. within typically $3.5-4 \AA$ ). This assumption has also been separately supported by several molecular dynamics simulations. For techniques like $\mathrm{THz}$ spectroscopy which probe lower-frequency, more collective motions involving many waters and possibly also the biomolecule, it has been suggested that the dynamical perturbation can extend up to $18 \AA$ from a protein surface. ${ }^{22}$ Among the techniques directly probing water dynamics, a final subgroup includes elastic and quasi-elastic neutron scattering, ${ }^{23,24}$ which probe the displacement of individual water hydrogen atoms, and employ solutions with a high concentration of biomolecules. The advantage is that all water molecules belong to a hydration shell, but the simple twostate description's validity becomes questionable: additional non-linear effects induced at those very high concentrations when a water molecule can become simultaneously affected by several solute molecules.

A second group of techniques shift their perspective: instead of directly probing the properties of water, they adopt the biomolecule viewpoint and monitor a property of the biomolecule affected by the solvent dynamics. Perhaps the outstanding example of this approach is provided by time-dependent Stokes' shift (TDSS) experiments. ${ }^{4}$ In its pump-probe approach, a first ultrafast (sub-ps) laser pulse electronically excites a chromophore within the biomolecule (this chromophore can either be an extrinsic synthetic dye or an intrinsic group already present in the biomolecule, like the tryptophan aromatic side-chain in proteins). This electronic transition produces a change in the 
chromophore charge distribution, and by the Franck-Condon principle, the surrounding hydration structure that was adapted to the electronic ground state charge distribution is now out of equilibrium. The water solvent thus starts relaxing towards a new equilibrium structure adapted to the electronic excited state. As the solvent relaxes, the Stokes' shift, i.e. the difference between the absorption and fluorescence energies, increases from zero to its equilibrium value. A second ultrafast pulse can be used to measure the emission energy through a stimulated emission process, and scanning the time delay between the pump and probe pulses provides the full time-resolved solvent relaxation (which is why these are often called "solvation dynamics" experiments).

This technique requires neither high solute concentrations nor any assumption on the number of water molecules affected. However, in contrast to e.g. NMR which probes the reorientation of a single water molecule and neutron scattering which follows the displacements of individual water hydrogen atoms, TDSS is sensitive to collective motions affecting several water molecules and possibly of the protein itself, since they all influence the chromophore's fluorescence energy. On the other hand, these types of water dynamics could be most relevant in aqueous chemical reactions where a reacting solute rapidly changes its charge distribution.

\section{Is the biomolecular hydration layer viscous or labile?}

While it is certainly reasonable to expect a biomolecular interface to perturb the dynamics of water molecules in the hydration shell, a key question to which the answer is not immediately obvious relates to the sign of this perturbation: is water faster or slower in the shell than in the bulk? The two important reference points here are on the one hand the dynamics in the bulk ( $2.5 \mathrm{ps}$ for water reorientation at room temperature) and on the other hand the dynamics of the biomolecule itself, whose typical tumbling time lies in the nanosecond range. Perhaps the more dramatic of possibilities would be if water molecules were so retarded by the interface that their dynamics relative to the biomolecule was slower than the biosolute tumbling; this would imply that the 
biomolecule moved together with an intact hydration layer.

We now briefly review some important measurements of the hydration layer dynamics, starting with the historical experiments that were erroneously interpreted as indicating the presence of an extremely slow, almost ice-like, layer around proteins and DNA, and finishing with more recent experiments which clearly suggest that the hydration layer is more moderately retarded, although the magnitude of the slowdown is still debated.

The first studies that suggested the presence of a rigid water layer around proteins estimated the typical size of the protein reorienting in solution from its tumbling time $\tau_{\text {reor }}$ determined by NMR through the Debye-Stokes-Einstein (DSE) equation

$$
\tau_{\text {reor }}=\frac{4 \pi \eta a^{3}}{3 k_{B} T}
$$

where $\eta$ is the solvent shear viscosity, $\mathrm{T}$ is the temperature and $a$ is the the radius of the protein approximated by a sphere. We need to know that this equation relies on several assumptions. First, the motion is assumed to be diffusive, i.e. protein motion occurs through a sequence of tiny uncorrelated steps; this is verified for proteins since the characteristic timescale for the protein angular displacement is much longer than that of the relaxation of its (angular) momentum. Second, the DSE equation assumes a continuum hydrodynamic description, in which the water solvent is treated as being continuous rather than molecular, using the equations that govern the dynamics of continuous fluids. The protein sizes obtained via DSE were systematically larger than those coming from crystal structures, a result which was explained by the presence of a frozen water layer moving together with the protein, thus increasing its hydrodynamic radius. ${ }^{3,25}$ The flaw in this argument was subsequently revealed when it was shown that the simple continuum hydrodynamic picture is not valid for the protein hydration layer. Explicitly accounting for the protein surface roughness, for the dielectric friction between the protein and the water solvent, and for the fact that the hydration layer is not bulk-like invalidates the picture of an ice-like layer. ${ }^{3,25}$

A second indication of the presence of very slow water molecules around proteins 
came from dielectric relaxation spectroscopy experiments. This technique probes the relaxation of the total dipole moment of the aqueous protein solution and the spectrum obtained in the frequency domain reveals the characteristic timescales of the different motions involved in the relaxation of this dipole. The presence of a nanosecond timescale (called the $\delta$ step) in the dielectric relaxation spectrum was traditionally explained by the very strong retardation of typically half the hydration shell water molecules. However, more recent work ${ }^{26}$ suggests that it instead originates from slow rearrangements of the hydration layer which are induced by slow protein conformational motions; that is to say, the intrinsic water dynamics are rapid (and not 'frozen'), but they respond to slow protein dynamics.

In a further older indication of slow hydration shell dynamics around proteins and DNA, NMR experiments employing a magnetization transfer between water and protein protons through the nuclear Overhauser effect (NOE) were at first interpreted as showing that water dynamics occurs on the $300 \mathrm{ps}-1 \mathrm{~ns}$ timescale in the hydration shell. However, subsequent work showed that these studies did not properly account for labile protein hydrogens and for long-range couplings of protein protons with bulk water, ${ }^{3}$ and that NOE measurements are actually more sensitive to bulk dynamics than to hydration shell dynamics.

As our account above indicates, an accurate perception of the dynamics of biomolecular hydration layers has proved challenging in the past. Fortunately, more recent studies have helped clarify these dynamics, as now recounted.

A much improved characterization of biomolecular hydration dynamics has been provided by a variant of NMR called magnetic relaxation dispersion (MRD). In MRD, the longitudinal spin relaxation rate is measured at different resonance frequencies to yield the Fourier-transform of the water orientation time-correlation function (i.e. the spectral density). ${ }^{3,27}$ While each measurement is averaged over the entire hydration shell, the frequency dependence obtained by MRD provides some information on the distribution of relaxation times within the shell; this ability is especially valuable in situations where the shell dynamics is heterogeneous, as it certainly is next to biomolecules as we will see in Section 5. An MRD study of a series of globular pro- 
teins showed that the room temperature rotational dynamics within the hydration layer is only moderately retarded compared to the bulk water situation, by a factor of approximately $2-3 .{ }^{27}$ This conclusion was supported both by the finding of similar values for the slowdown in water reorientation in molecular dynamics simulations ${ }^{20,28}$ and by a separate NMR determination of a retardation factor of 3 for the water translational dynamics. ${ }^{29}$ No such detailed study exists for DNA, but MRD also concluded that there is no long-lived water molecule tumbling together with DNA. ${ }^{30}$

TDSS experiments have provided the first time-resolved investigations of protein and DNA hydration dynamics. ${ }^{4,31}$ Comparison of the solvation dynamics decays of the same chromophore isolated in aqueous solution and at the biomolecular interface showed that the solvation dynamics is slower within the hydration layer. In addition, while the TDSS decay exhibits a single, sub-picosecond component in bulk water, it is biphasic in the biomolecular case, with a first, picosecond component and a second, slower timescale on the order of a few tens of picoseconds (20-200 $\mathrm{ps}^{32}$ ). The faster decay was assigned to bulk-like labile water molecules, while the slower decay was interpreted as due to water molecules strongly interacting with the biomolecular interface. ${ }^{4,31}$ The origins of these two decays have been much debated. ${ }^{3,31,33}$ In a focus on the slow component in the protein case, both $\mathrm{NMR}^{27}$ and MD simulations ${ }^{28}$ find that only a very small fraction of the water molecules present in the hydration layer exhibit reorientation dynamics slower than 10 ps. Furthermore, different NMR approaches measuring the water rotational ${ }^{27}$ and translational ${ }^{29}$ dynamics both indicate an average retardation factor of three within the protein hydration layer compared to the bulk. All this suggests that the slow $>10$ ps decay observed in TDSS experiments likely originates from coupled protein-water motions and water molecules displaced by slow conformational rearrangements of the protein, rather than from any intrinsically slow motions of the waters. ${ }^{33-35}$ Similar results have been obtained for DNA hydration dynamics. A slow component is observed in the TDSS results ${ }^{4}$ and based on molecular dynamics simulations it has been argued that it arises from a slow DNA conformational motion induced by the chromophore. ${ }^{36}$

Many other techniques have been applied to the study of protein hydration dynam- 
ics. Two-dimensional infrared spectroscopy with a local carbonyl probe has also determined a modest slowdown factor - approximately 2 - for H-bond dynamics within the hydration layer. ${ }^{37}$ Techniques like optical Kerr-effect spectroscopy ${ }^{38,39}$ instead probe collective water dynamics (e.g. the relaxation of the system polarization) and have measured slightly larger slowdown factors between 7 and 9 , which remain to be explained.

The idea of an ice-like hydration shell moving rigidly with the protein has now been completely discounted. However, the exact magnitude of the slowdown of water dynamics in biomolecular hydration shells remains a topic of debate. Different techniques appear to give different results, which may be explained by the different dynamics they probe.

\section{How heterogeneous is water dynamics within the shell?}

As we detailed in the preceding section, it is clearly established that the presence of a biomolecular interface perturbs the dynamics of water compared with the bulk situation, even though the magnitude of the effect might be debated. A natural question to ask then is whether this perturbation is identical everywhere within the hydration layer or whether some sites perturb water more significantly than do others. A priori, the latter seems more likely: the exposed surface of a biomolecule is indeed a very heterogeneous interface, both chemically and topologically. A wide variety of different chemical groups are present: charged, polar and apolar groups, H-bond acceptors and donors. Even beyond this, the shape of the exposed surface is usually rough on the molecular level, with a succession of pockets, protrusions and grooves.

Molecular dynamics simulations provide a first indication that water dynamics is not homogeneously affected by the biomolecular interface. If we consider for example the rotational dynamics (which is probed e.g. by NMR), the reorientation time of a given water molecule is quantitatively defined from the time-correlation function of the molecular orientation. ${ }^{40}$ This function compares the molecular orientations at an 
initial time and after a given delay, and tracks how quickly a water molecule loses the memory of its initial orientation. The reorientation time can be defined as the timeintegral of this decay. ${ }^{40}$ When this is computed for all the water molecules initially present in the hydration layer of a protein or a DNA strand, it exhibits a pronounced non-exponential decay ${ }^{28}$ which strongly suggests the presence of a distribution of sites with different dynamics.

Identifying which sites cause a greater (or smaller) perturbation requires a siteresolved mapping of the dynamics of water around the biomolecule. This has remained a long-standing goal: obtaining detailed, spatially resolved information on hydration layer water dynamics is extremely challenging for all experimental techniques. What is the nature of the difficulties? While NMR can provide a valuable determination of the dynamical perturbation factor, this information is intrinsically averaged over the entire hydration shell. If a given functional, i.e. a given "shape", is assumed for the distribution of relaxation times, MRD can be used to determine the distribution's parameters, but it cannot identify the locations on the biomolecular surface responsible for the distribution's different components. Recent promising NOE-NMR work ${ }^{41}$ has obtained a spatial mapping of water dynamics, but at a cost: it requires specific conditions and an encapsulation of the protein in a reverse micelle. As discussed below in Sec. 8, confinement has a strong, probably non-uniform, effect on hydration layer water dynamics.

The first site-resolved mapping of solvation dynamics within a biomolecular hydration shell was obtained by performing TDSS experiments on a series of different protein mutants, where the fluorescent chromophore's location was systematically scanned over the protein's exposed surface. ${ }^{32}$ Those studies showed that the characteristic biphasic decay of the TDSS (mentioned in the previous section) is conserved in all the mutants, and the main observation is that the decay timescales (and thus hydration dynamics) tend to be shorter next to hydrophobic and/or flexible groups. ${ }^{32}$

Very recently, a new ultrafast infrared technique was also shown to probe water H-bond dynamics within a protein hydration layer with both a femtosecond time resolution and a spatial resolution of approximately a few Angströms. This technique 
employs a metal carbonyl probe whose vibrational dynamics is sensitive to the surrounding water dynamics. Monitoring the carbonyl vibrational frequency via twodimensional infrared spectroscopy thus provides a measure of the local water H-bond dynamics. This probe can in principle be attached to different locations on the protein surface. In the technique's first results, two probe locations on lysozyme were compared and it was shown that water frequency dynamics is almost bulk-like next to exposed, flexible groups and more retarded in more constraining hydrophobic protein environments. ${ }^{37}$

Achieving a detailed site-resolution is clearly experimentally difficult, but molecular dynamics simulations is a tool which is perfectly suited for that purpose: for each protein or DNA site, one can determine the reorientation time of water molecules initially located next to it. The resulting distributions of relaxation times within a protein hydration layer have been determined in different studies. For a major fraction of the hydration shell (more than 75\%), the retardation factor compared to the bulk is moderate and lies between 1 and 3. The distribution also includes a long tail at larger slowdown values which results from the remaining $25 \%$ fraction of the hydration layer and which can be fit by a power law. ${ }^{28,42}$ Figure 1 shows the resulting mapping on the exposed surfaces of three different globular proteins in their native state. ${ }^{28}$ Even though the exposed surfaces are chemically heterogeneous and topologically rough, the water dynamics is fairly similar for most of the exposed surface. The sites where water is more retarded are mainly specific and buried locations, e.g. clefts and pockets.

In summary, clear progress is being made in the detailed characterization of hydration dynamics within biomolecular hydration shells and their site-resolved mapping. There remains however a central challenge to identify the protein features which actually determine the dynamical perturbation of water. Before we examine this question in the next section, we should also briefly mention that in addition to the spatial heterogeneity we have just described, a temporal heterogeneity can also be caused by biomolecular conformational fluctuations. These motions are slow compared to typical hydration shell dynamics and they can affect the dynamics of water molecules for 


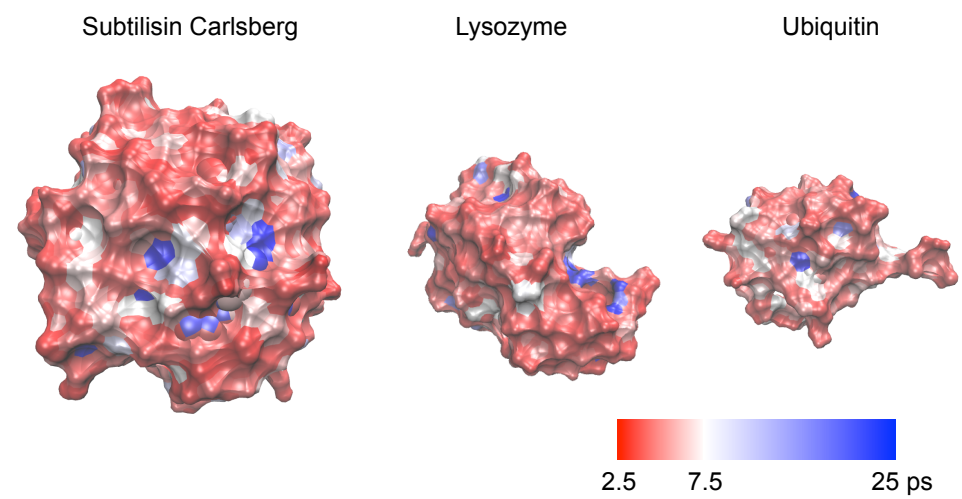

Figure 1: Maps of water reorientation time around a series of globular proteins. ${ }^{28}$

example by modulating the width of a protein cleft or that of a DNA groove.

\section{What is the molecular origin of the dynamical pertur- bation induced by the biomolecule on water? chem- istry vs. topology}

Unfortunately, the main properties responsible for the hydration shell perturbation by the protein cannot be clearly identified through a simple inspection of the perturbation map described in Sec. 5. Likely candidates include the local topology of the biomolecule's exposed surface and the chemical nature of the different residues and nucleotides (although other factors including e.g. the protein's secondary structure have been suggested). It is a challenge to separate the topological effect from the energetic factor, i.e. to separate the effect due to the surface's rugged nature from the effect which arises from the different interaction energies of water with the surface residues and which depends on their chemical identity. In this effort, a molecular dynamics study ${ }^{43}$ showed that the dynamics of water computed next to a protein in the regular conditions is slower than those obtained when all the protein partial charges are removed, thus effectively turning the protein into a large hydrophobic, rough solute. This approach, while ingenious, is unfortunately limited by the very strong perturba- 
tion to the system; for example the hydration layer structure is completely reorganized when the protein is made apolar. ${ }^{43}$

The previously elusive connection between protein properties and hydration dynamical perturbation was recently determined through a molecular jump description of water H-bond dynamics developed by some of us. We now pause to describe briefly this extended jump model, which successfully describes water H-bond dynamics next to a wide range of solutes. This novel picture had its origin in the finding that bulk water molecules mainly reorient through sudden, large-amplitude angular jumps when a water hydroxyl $(\mathrm{OH})$ group trades H-bond acceptors (Fig. 2.a). ${ }^{44}$ Further, this trading of acceptors can be regarded as a chemical reaction. An additional, usually minor, contribution arises from the slower tumbling of the molecular frame for an $\mathrm{OH}$ involved in an intact $\mathrm{H}$-bond between $\mathrm{H}$-bond acceptor exchanges. We refer the interested reader to Ref. ${ }^{40}$ for further details of the model and its applications to various simple solutes. When applied to describe a typical protein hydration layer, the model predicts a distribution of water reorientation times in good agreement with the directly computed distribution, thus suggesting that this extended jump model may be used to analyze hydration shell dynamics. Before elaborating on this, we need to pause and detail the molecular factors which explain the perturbation of water dynamics by the presence of a solute; the jump picture's chemical reaction interpretation plays a central role here.

The jump rate constant depends on two key local solute features, which reflect the topological and chemical aspects we have highlighted earlier. Each of these features can be quantified, providing a multiplicative factor - associated with the transition, or activated, state of the reaction - which affects the rate. ${ }^{40}$ The first feature - which is topological and induced by any type of solute and interface - results from the partial hindrance of the approach of a new water H-bond partner compared to the bulk situation; this leads to a slowdown in the jump rate, which can be quantified by the transition-state excluded volume factor $\rho_{\mathrm{V}} \cdot{ }^{40,45}$ The second feature results from the strength of the initial H-bond; compared to the bulk situation, it accelerates the jump rate if the initial bond is weaker than a water-water H-bond, and slows the rate if the bond is stronger. The effect can be quantified by the transition-state hydrogen-bond 
strength factor $\rho_{\mathrm{HB}} .{ }^{40}$ Then the overall perturbation factor $\rho$ relative to the bulk results from the combination of these two factors, $\rho=\rho_{\mathrm{V}} \rho_{\mathrm{HB}}$. In the protein and DNA context, this analysis clearly separates the respective contributions from the local topology $\left(\rho_{\mathrm{V}}\right)$ and from the strength of the interaction between water and the biomolecular sites $\left(\rho_{\mathrm{HB}}\right)$.

Figure 2.b schematically summarizes the key effects expected for the three main types of sites found at a biomolecular interface: hydrophobic groups, H-bond donors and H-bond acceptors, now discussed in turn. Hydrophobic groups only affect H-bond jump dynamics by hindering the approach of a new water H-bond acceptor through an excluded volume effect. H-bond donors can form bonds of different strengths, but these bonds only act on the water oxygen around which the angular jump occurs, and the resulting torque's influence on the $\mathrm{OH}$ reorientation is negligible. Thus H-bond donors, like hydrophobic groups, perturb water dynamics mainly via their excluded-volume effect. Finally, H-bond acceptor groups can influence water H-bond dynamics via both their excluded-volume effect and the strength of the H-bond formed with water.

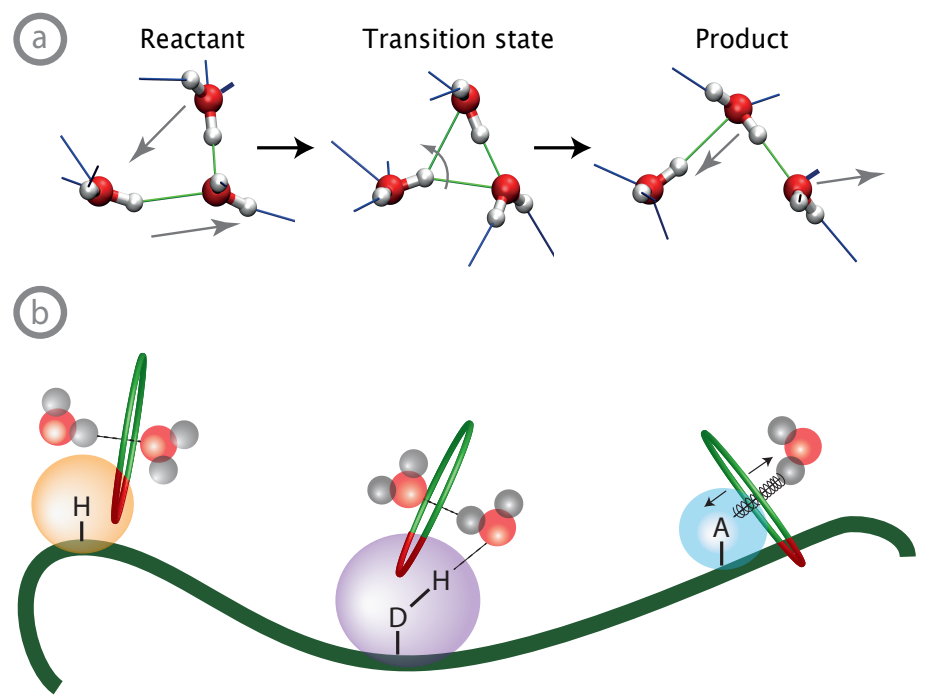

Figure 2: a) Molecular jump mechanism. b) Schematic figure with a protein interface and the three types of sites, respectively hydrophobic, H-bond acceptor and H-bond donor, together with a pictorial representation of the types of perturbation they induce on water dynamics (excluded volume and $\mathrm{H}$-bond strength factors). 
The extended jump picture together with the excluded-volume and H-bond strength factors influencing the jump kinetics that we have just described provides a simple analysis of the distribution of perturbation factors within the hydration shell. An attractive asset of this approach is that it depends only very weakly on the force-field adopted because it relies on the identification of the physical mechanisms of the hydration shell dynamics perturbations. For a series of recently studied globular proteins, ${ }^{28,46}$ approximately three quarters of the hydration layer waters experience a moderate perturbation factor (between 1 and 3) due to an excluded volume effect, i.e. to the protein surface's local topology; locally convex sites (i.e. protrusions) lead to perturbation factors below 2, while locally concave sites (i.e. pockets, clefts, crevasses) lead to factors above 2. These waters essentially correspond to those molecules lying next to hydrophobic and $\mathrm{H}$-bond donor groups. The remaining fraction of waters experiencing a greater slowdown arises mostly from deeply buried H-bond acceptor groups of moderate interaction strength like the backbone carbonyl groups, and from strong $\mathrm{H}$-bond acceptor groups like the carboxylates present in the glutamate and aspartate residues. The dominant importance of topological factors over the amino-acid composition in determining the hydration shell perturbation has received further support from experimental and computational studies which suggest that mutants exhibit very similar hydration dynamics. ${ }^{46,47}$

The two main features identified as determining the biomolecule's hydration dynamics are local factors, namely the local biomolecular surface topology and the nature of the chemical groups interacting with water molecules. However, these local factors can be affected by longer-range properties of the protein sequence. Knowing the identity of the amino-acid closest to the water molecule under consideration is not sufficient to determine these local factors. For example the local protein topology is partly determined by the protein's secondary structure which depends on the whole sequence of amino-acids. While an isolated amino-acid always displays a convex exposed surface, the local topology when considered within a protein pocket can become concave, thus affecting differently the water dynamics. This explains why the hydration dynamics next to a given amino-acid was measured to change with the amino-acid location 
on a protein surface. ${ }^{28}$ Other studies have also suggested that water dynamics within biomolecular hydration layers are affected by much more collective factors, including the presence of a percolating network of H-bonds within the hydration layer, ${ }^{7,48}$ or the existence of a glassy behavior involving highly collective motions within the layer. ${ }^{7}$

\section{How does the perturbation change with temperature?}

All of the results and arguments discussed so far apply to ambient temperature (usually taken to be $300 \mathrm{~K}$ ), the most common relevant temperature for such biological systems as proteins and DNA. However, life is not limited to such gentle conditions. Some living organisms (extremophiles) thrive in extreme environments: psychrophilic organisms can survive the very cold temperatures found in e.g. polar regions, while thermophiles such as the archeae found next to hydrothermal vents have adapted to very hot environments. These facts clearly furnish a strong biochemical motivation to understand how the ideas developed for room temperature hydration dynamics can be extended.

As we described in Section 4, it is now clearly established that at room temperature a biomolecule induces a slowdown in the surrounding water dynamics. However, recent NMR-MRD results reveal that this perturbation changes dramatically with temperature. ${ }^{27}$ These experiments, performed on a series of globular proteins over the broad temperature range $238 \mathrm{~K}$ to $290 \mathrm{~K}$, indicate that the protein-induced dynamical perturbation factor is strongly affected by the temperature. Strikingly, when the system is cooled below approximately $260 \mathrm{~K}$, the hydration shell dynamics slowdown factor drops very rapidly and approaches unity (see Fig. 3). This implies that the average protein shell water dynamics becomes similar to that in the bulk, intriguingly suggesting that the protein's dynamical perturbation disappears. We hasten to note that at these very low (approximately $240 \mathrm{~K}$ ) temperatures, water is in a supercooled state and such conditions are probably not biologically relevant. On the other hand, experimental results on such a broad temperature range are extremely valuable for testing and refining the descriptions used at the milder temperatures where biological systems function. We 
proceed in this spirit.

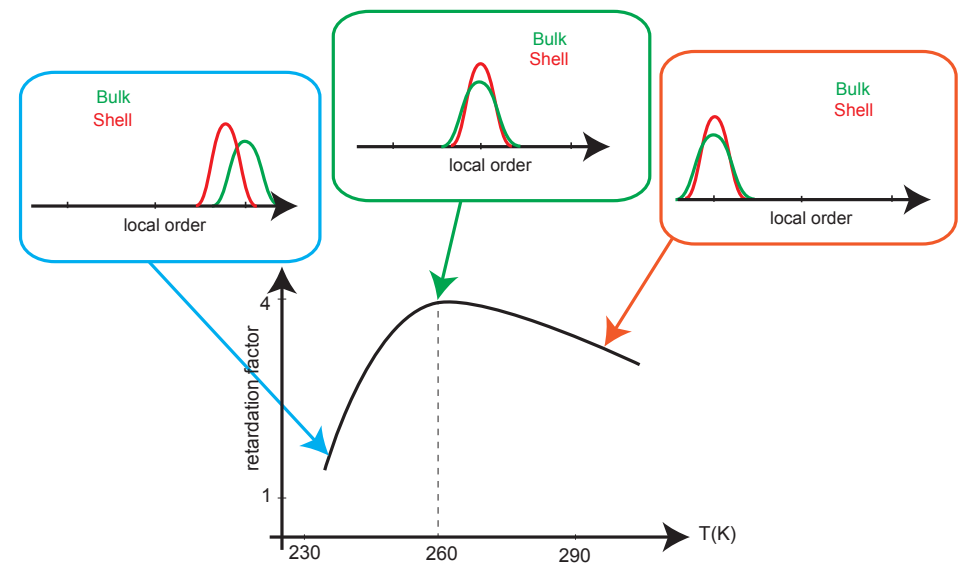

Figure 3: Schematic representation of the perturbation factor's non-monotonic temperature dependence ${ }^{27}$ together with the local order distributions in the bulk and in the shell at three temperatures.

Other NMR-MRD measurements ${ }^{49}$ provide an instructive comparison in a simpler case. Water's dynamical perturbation within the hydration shell of small, mostly hydrophobic solutes is found to exhibit the same type of temperature-dependence as described for proteins. Although a protein is obviously much more complex and heterogeneous than a small hydrophobic solute, we saw in Sec. 6 that for most protein hydration layer water molecules, the main perturbation arises from an excluded volume effect, exactly like for waters next to a hydrophobic solute. Consequently, understanding hydrophobic hydration dynamics' temperature dependence should provide the key factor governing that of the protein hydration dynamics.

The slowdown in a hydrophobic group's surrounding water dynamics stems, as just mentioned, from an entropic excluded volume effect, ${ }^{45}$ and this would not be expected to depend significantly on temperature. A probable explanation of the decrease in the slowdown factor with decreasing temperature comes from the progressive structuring of water at low temperature. In liquid water, the local structure surrounding a water molecule continuously fluctuates due to thermal motions. However, the average structure becomes more tetrahedral upon cooling, approaching the ideal ice structure, and this increases the free energy barrier for H-bond jumps since it is more difficult to dis- 
rupt a better organized local structure. ${ }^{50}$ The key point is that when the temperature decreases, local water structures shift in different ways in the bulk and in the shell ${ }^{49}$ because the solute interface's presence partly hinders the tetrahedral structuring that is preserved in the bulk. As a result, the jump free energy barrier does not increase as much in the hydration layer as in the bulk. Consequently, the slowdown factor's dramatic drop at very low temperature is due not so much to the solute-induced perturbation's vanishing as it is to the bulk water dynamics' slowdown due to a local structure ordering. For proteins, our picture for hydrophobic solutes should be further refined because of the additional complexity arising from the great chemical heterogeneity of the protein's exposed surface. In particular, sites with different interaction energies with water should lead to local water dynamics with different Arrhenius activation energies.

The discussion above has focused on the hydration shell behavior and its temperature dependence. We stress that it is distinct from other phenomena that may occur at low temperature and which affect the protein itself. These include for example the "dynamical transition" observed close to $200 \mathrm{~K}$, where hydrated proteins have been suggested to pass from a frozen state to a regime where collective motions appear. ${ }^{51-53}$ Another example is cold denaturation, which designates the loss of the protein compact folded structure at low temperature. ${ }^{54}$

\section{Can one go beyond the dilute globular protein case?}

We have so far focused on the - already difficult - ideal case of a single biomolecule surrounded by neat water in a dilute aqueous solution. However, as illustrated in Sec. 2, situations where biomolecular hydration dynamics can play an important biological role often involve more complex environments. This is for example the case within biological cells in the cytoplasm where a biomolecule is surrounded not only by water molecules but also by other solutes such as ions, sugars and osmolytes. It also applies to biomolecules used in such technological applications as the storage of biomolecules at high concentration and drug delivery by encapsulation. We now consider how the concepts developed in the preceding sections can be extended to those situations. We 
focus on three main aspects - impact of confinement, the consequences of unfolding, and the effect of ions and denaturants on biomolecular hydration dynamics - before finally discussing some recent experimental results on hydration dynamics in biological cells.

Confinement. It is well established that confinement slows down water dynamics. For example, an increasing retardation factor in reorientation dynamics for neat water confined within reverse micelles of decreasing sizes was measured by ultrafast infrared spectroscopy. ${ }^{55}$ The impact of confinement for protein hydration dynamics was recently investigated by two-dimensional infrared spectroscopy (see Sec. 4) on a protein in water-glycerol solutions. ${ }^{37}$ Since water molecules segregate at the protein interface, increasing the glycerol fraction leads to the protein hydration layer's increasing confinement. Going from neat water to a $80 \%$ glycerol volume fraction leads to a three-fold slowdown in water dynamics. With the jump picture detailed in Sec. 6, this slowdown upon confinement can be explained by the increased difficulty of motions required for a water molecule to find a new H-bond partner. Confinement was also recently exploited to reduce undesirable contributions to the NMR-nuclear Overhauser effect signal and to access a detailed mapping of hydration dynamics around a protein encapsulated within a reverse micelle. ${ }^{41}$ Measured hydration timescales ranged from picoseconds to nanoseconds, suggesting the presence of clustering of sites with similar hydration dynamics. These results are especially interesting in the context of hydration dynamics at protein/membrane and protein/protein interfaces; however, since the measured slowdown is much greater than that induced separately by the reverse micelle ${ }^{55}$ or the protein, ${ }^{27}$ it is not yet clear whether the measured map reflects only the protein's influence and whether the slowdown induced by the reverse micelle is uniform throughout the protein surface.

Unfolding and misfolding. As discussed in Sec. 2, water dynamics within the protein hydration layer is suggested to play a key role in protein folding. ${ }^{6}$ However, we have not yet discussed the fact that hydration layer dynamics is also strongly affected by the large change in protein topology occurring during folding or unfolding. When a protein unfolds from a compact globular structure to an extended random coil, the 
pockets and clefts formerly present in the folded structure tend to disappear. The topological excluded-volume factor will thus tend to decrease, since fewer water molecules reside in locally concave environments with a large slowdown factor. This is confirmed by NMR experiments ${ }^{47}$ and by molecular dynamics simulations, ${ }^{46}$ both of which find a weaker perturbation factor around the unfolded protein compared to its folded form.

Ions and denaturants. A wide variety of protein behavior, including protein folding, enzymatic activity, precipitation and crystallization, is strongly affected by the presence of ions. ${ }^{56}$ For example, Hofmeister discovered more than a century ago that some ions tend to precipitate egg white proteins, while some others increase their solubility. A traditional explanation rationalizes these phenomena by invoking these ions' effect on the protein hydration shell. In this picture, the protein and the ions would compete for hydration, and ions which interact strongly and favorably with water (so-called "structure-makers") would tend to sequester water molecules, leading to the protein's dehydration and precipitation. The action of ions on the protein's stability would thus be water-mediated. A similar picture has been suggested for the action of denaturants and of folding chaperones. However, this picture is not clearly supported by experiments; indeed an increasing number of results support a description where ions directly interact with some specific protein sites in the presence of water, ${ }^{57}$ and the jump model approach might contribute to establishing the exact role played here by water.

Biological cells. In further progress towards more complex but also more biologically relevant systems, several studies have investigated water dynamics within living cells of different microorganisms: E. Coli and a cell from a halophilic organism living in the Dead Sea (where the salt concentration is very high). An NMR study ${ }^{58}$ of water in these cells determined the water reorientation time and concluded that in both cells hydration dynamics is more retarded than that measured next to dilute proteins; the average slowdown factor of approximately 15 was assigned to the larger fraction of confined hydration sites in the cell. The salt concentration within the cell was not measured to have a significant impact on the hydration dynamics. In striking contrast, a neutron scattering study ${ }^{59}$ measuring the water translational dynamics concluded that while water dynamics is almost bulk-like in E. Coli, it is retarded in the halophilic cell 
by the huge factor 250 . The origin of the discrepancy between these two sets of conclusions is not clearly established. While it is conceivable that some solutes may retard the water translational dynamics without much affecting the reorientation, a jump model analysis of water dynamics in concentrated solutions of amphiphiles suggests ${ }^{60}$ that this decoupling is probably not sufficient to explain the magnitude of the gap.

\section{Concluding remarks}

After decades of effort and the combination of different experimental and theoretical approaches, a molecular understanding of biomolecular hydration dynamics is emerging. Replacing the historical descriptions of the hydration shell as an ice-like layer, the currently largely accepted perspective pictures the shell as being composed of water molecules that are moderately retarded compared to the bulk. Several recent studies have characterized the dynamical heterogeneity within proteins' hydration shells and suggested which molecular factors determine the extent of the perturbation. Another arena where similar ideas can also be fruitfully applied is the understanding of DNA hydration shell dynamics which has been comparatively less studied so far. ${ }^{7}$ These recent achievements pave the way for new exciting developments in this field. Those include for example an improved description of the connection between the dynamical aspects of hydration detailed here and the thermodynamical properties ${ }^{8}$ which are crucial for the biomolecular functioning. This should also bring a molecular-level understanding of the effect of hydration shell dynamics on biomolecular function, which could be exploited in myriad ways, such as in the possible tuning of enzymatic activity and specificity through a proper choice of solvent conditions. ${ }^{10}$

\section{Acknowledgments}

The research leading to these results has received funding from the European Research Council under the European Union's Seventh Framework Program (FP7/20072013)/ERC grant agreement no 279977. JTH acknowledges support via NSF grant 
CHE-1112564.

\section{References}

[1] I. D. Kuntz and W. Kauzmann, Adv. Prot. Chem., 1974, 28, 239-345.

[2] J. A. Rupley and G. Careri, Adv Prot Chem, 1991, 41, 37-172.

[3] B. Halle, Philos Trans R Soc Lond B Biol Sci, 2004, 359, 1207-23; discussion $1223-4,1323-8$.

[4] S. K. Pal and A. H. Zewail, Chem Rev, 2004, 104, 2099-2124.

[5] B. Bagchi, Chem Rev, 2005, 105, 3197-219.

[6] Y. Levy and J. N. Onuchic, Annu Rev Biophys Biomol Struct, 2006, 35, 389-415.

[7] P. Ball, Chem Rev, 2008, 108, 74-108.

[8] L. Wang, B. J. Berne and R. A. Friesner, Proc Natl Acad Sci, 2011, 108, 13261330.

[9] P. Setny, R. Baron, P. Michael Kekenes-Huskey, J. A. McCammon and J. Dzubiella, Proc Natl Acad Sci U S A, 2013, 110, 1197-202.

[10] A. M. Klibanov, Nature, 2001, 409, 241-6.

[11] R. V. Dunn and R. M. Daniel, Philos Trans R Soc Lond B Biol Sci, 2004, 359, 1309-20; discussion 1320, 1323-8.

[12] M. Grossman, B. Born, M. Heyden, D. Tworowski, G. B. Fields, I. Sagi and M. Havenith, Nat Struct Mol Biol, 2011, 18, 1102-8.

[13] S. C. L. Kamerlin and A. Warshel, Proteins, 2010, 78, 1339-75.

[14] B. Nguyen, S. Neidle and W. D. Wilson, Acc Chem Res, 2009, 42, 11-21.

[15] A. Mukherjee, R. Lavery, B. Bagchi and J. T. Hynes, J Am Chem Soc, 2008, 130, $9747-55$. 
[16] L. Szyc, M. Yang, E. T. J. Nibbering and T. Elsaesser, Angew Chem Int Ed Engl, 2010, 49, 3598-610.

[17] J. L. Silva, T. C. R. G. Vieira, M. P. B. Gomes, A. P. A. Bom, L. M. T. R. Lima, M. S. Freitas, D. Ishimaru, Y. Cordeiro and D. Foguel, Acc Chem Res, 2010, 43, 271-9.

[18] D. N. Lebard and D. V. Matyushov, Phys Chem Chem Phys, 2010, 12, 15335-48.

[19] F. Garczarek and K. Gerwert, Nature, 2006, 439, 109-12.

[20] M. Marchi, F. Sterpone and M. Ceccarelli, J Am Chem Soc, 2002, 124, 67876791.

[21] V. P. Voloshin, N. N. Medvedev, M. N. Andrews, R. R. Burri, R. Winter and A. Geiger, J Phys Chem B, 2011, 115, 14217-14228.

[22] B. Born, S. J. Kim, S. Ebbinghaus, M. Gruebele and M. Havenith, Faraday Discuss., 2009, 141, 161-173.

[23] M. C. Bellissent-Funel, J. M. Zanotti and S. H. Chen, Faraday Discuss., 1996, 103, 281-294.

[24] D. Russo, G. Hura and T. Head-Gordon, Biophys J, 2004, 86, 1852-62.

[25] B. Bagchi, Chem Phys Lett, 2012, 529, 1-9.

[26] A. Oleinikova, P. Sasisanker and H. Weingärtner, J Phys Chem B, 2004, 108, $8467-8474$

[27] C. Mattea, J. Qvist and B. Halle, Biophys J, 2008, 95, 2951-63.

[28] F. Sterpone, G. Stirnemann and D. Laage, J Am Chem Soc, 2012, 134, 4116-9.

[29] R. G. Bryant, C R Phys, 2010, 11, 128-135.

[30] V. P. Denisov, G. Carlström, K. Venu and B. Halle, J Mol Biol, 1997, 268, 118-36.

[31] D. Zhong, S. K. Pal and A. H. Zewail, Chem Phys Lett, 2011, 503, 1-11. 
[32] L. Zhang, L. Wang, Y.-T. . T. Kao, W. Qiu, Y. Yang, O. Okobiah and D. Zhong, Proc Natl Acad Sci, 2007, 104, 18461-18466.

[33] A. A. Golosov and M. Karplus, J Phys Chem B, 2007, 111, 1482-90.

[34] T. Li, A. A. Hassanali, Y.-T. T. Kao, D. Zhong and S. J. Singer, J Am Chem Soc, 2007, 129, 3376-82.

[35] B. Halle and L. Nilsson, J Phys Chem B, 2009, 113, 8210-3.

[36] K. E. Furse and S. A. Corcelli, J Phys Chem Lett, 2010, 1, 1813-1820.

[37] J. T. King and K. J. Kubarych, J Am Chem Soc, 2012, 134, 18705-18712.

[38] N. T. Hunt, L. Kattner, R. P. Shanks and K. Wynne, J Am Chem Soc, 2007, 129, 3168-72.

[39] K. Mazur, I. A. Heisler and S. R. Meech, J Phys Chem A, 2012, 116, 2678-2685.

[40] D. Laage, G. Stirnemann, F. Sterpone, R. Rey and J. T. Hynes, Annu Rev Phys Chem, 2011, 62, 395-416.

[41] N. V. Nucci, M. S. Pometun and A. J. Wand, J Am Chem Soc, 2011, 133, 12326-9.

[42] A. E. Garcia and G. Hummer, Proteins: Struct., Funct., Genet., 2000, 38, 261272.

[43] F. Pizzitutti, M. Marchi, F. Sterpone and P. J. Rossky, J Phys Chem B, 2007, 111, 7584-7590.

[44] D. Laage and J. T. Hynes, Science, 2006, 311, 832-835.

[45] D. Laage, G. Stirnemann and J. T. Hynes, J Phys Chem B, 2009, 113, 2428-2435.

[46] O. Rahaman, S. Melchionna, D. Laage and F. Sterpone, Phys. Chem. Chem. Phys., 2013, 15, 3570-3576.

[47] J. Qvist, G. Ortega, X. Tadeo, O. Millet and B. Halle, J Phys Chem B, 2012, 116, 3436-3444. 
[48] I. Brovchenko and A. Oleinikova, Chemphyschem, 2008, 9, 2695-702.

[49] J. Qvist and B. Halle, J Am Chem Soc, 2008, 130, 10345-10353.

[50] G. Stirnemann and D. Laage, J Chem Phys, 2012, 137, 031101.

[51] D. Ringe and G. A. Petsko, Biophys Chem, 2003, 105, 667 - 680.

[52] M. Tarek and D. Tobias, Phys Rev Lett, 2002, 88, 138101.

[53] K. Wood, A. Frölich, A. Paciaroni, M. Moulin, M. Härtlein, G. Zaccai, D. J. Tobias and M. Weik, J Am Chem Soc, 2008, 130, 4586-7.

[54] C. F. Lopez, R. K. Darst and P. J. Rossky, J Phys Chem B, 2008, 112, 5961-5967.

[55] M. D. Fayer and N. E. Levinger, Annu Rev Anal Chem, 2010, 3, 89-107.

[56] Y. Zhang and P. S. Cremer, Annu Rev Phys Chem, 2010, 61, 63-83.

[57] K. B. Rembert, J. Paterová, J. Heyda, C. Hilty, P. Jungwirth and P. S. Cremer, J Am Chem Soc, 2012, 134, 10039-46.

[58] J. Qvist, E. Persson, C. Mattea and B. Halle, Faraday Discuss., 2009, 141, 131144.

[59] M. Tehei, B. Franzetti, K. Wood, F. Gabel, E. Fabiani, M. Jasnin, M. Zamponi, D. Oesterhelt, G. Zaccai, M. Ginzburg and B. Z. Ginzburg, Proc Natl Acad Sci U $S$ A, 2007, 104, 766-71.

[60] G. Stirnemann, F. Sterpone and D. Laage, J Phys Chem B, 2011, 115, 3254-3262.

\section{Biographies}

\section{Aoife C. Fogarty}

Aoife Fogarty was born in Ireland in 1987. She received her Master of Chemistry from the University of Edinburgh in 2010, and is currently a Ph.D student at Ecole Normale Supérieure and the University of Paris (UPMC) under the supervision of Damien 
Laage. Her thesis topic is the study of enzymatic reactions in organic solvents, and the perturbation of water dynamics by solutes or interfaces.

\section{Elise Duboué-Dijon}

Elise Duboué-Dijon was born in France in 1990. She graduated from the University of Paris (UPMC) and Ecole Normale Supérieure (ENS) in 2012 with a Master of Chemistry degree, specialized in physical and theoretical chemistry. She is currently working for a PhD at the ENS and UPMC under the supervision of Damien Laage. Her current research focuses on the influence of a protein on its hydration shell dynamics, as well as on the influence of solvent properties on enzyme catalysis.

\section{Fabio Sterpone}

Fabio Sterpone was born in Italy in 1973. He received his B.S. in Physics (1999) from University of Rome and his Ph.D. (2004) from University of Paris UPMC. He has occupied successive postdoctoral positions at University of Texas (Austin), HPC Caspur (Rome), and Ecole Normale Supérieure (Paris). His research actvity is focused on biomolecular and multi-scale simulations. He is currently a CNRS researcher at Institut de Biologie et Physico-Chimie (Paris).

\section{James T. Hynes}

James T. Hynes was born in Florida in 1943. He received his A.B. from Catholic University in 1965 and his Ph.D. from Princeton University with J. M. Deutch in 1969. After an NIH postdoctoral fellowship at MIT with Irwin Oppenheim, he joined the University of Colorado, Boulder faculty in 1971. He is currently Distinguished Professor of Chemistry and Biochemistry. Since 1999, he has also been CNRS Director of Research at ENS. His research focuses on reaction and allied dynamics in solution, biomolecules and at interfaces. 


\section{Damien Laage}

Damien Laage was born in France in 1975. He received his Ph.D. in 2001 from Ecole Normale Supérieure (ENS) and University of Paris (UPMC) with James T. Hynes and Monique Martin. He was a postdoctoral fellow at ETH Zürich with Michele Parrinello and in 2002 joined ENS where he and his group study chemical reactivity and spectroscopy in solutions and biochemical environments. In 2011, he received an ERC Starting Grant to study solvent effects in enzyme catalysis. 